\begin{tabular}{|c|c|c|c|c|c|}
\hline Revista Clío América & ISSN: 1909-941X & Vol. 10 & No. 20 & Julio - Diciembre de 2016 & 118 - 125 \\
\hline
\end{tabular}

\title{
Impacto de los programas de aportes y donaciones de almacenes de cadena de Cartagena sobre la imagen y reputación
}

\author{
Impact of contributions programs and donations from warehouses of chain of Cartagena on the \\ image and reputation
}

Resumen: El presente artículo es resultado de un estudio realizado a los almacenes de cadena de la ciudad de Cartagena para medir el impacto de los programas de aportes y donaciones sobre la imagen corporativa y reputación. Se hace primero un seguimiento de la discusión teórica reciente alrededor de los conceptos de RSE, aportes y donaciones, imagen corporativa y reputación. Luego se aplica un método cuantitativo y se formulan cuatro hipótesis para medir la incidencia de las variables acción social y relaciones con la comunidad sobre la imagen corporativa y reputación. La discusión teórica encontró que la RSE debe entenderse como una filosofía de gestión empresarial comprometida con el mejoramiento de las condiciones de vida de la sociedad en general. Adicionalmente, las pruebas de hipótesis confirmaron las diferencias conceptuales entre la imagen corporativa y reputación por encontrarse una relación directa y positiva entre las variables acción social y relaciones con la comunidad sobre la imagen corporativa y una relación no directa entre las variables acción social y reputación. Esto muestra que los programas de aportes y donaciones pueden contribuir a mejorar la imagen corporativa, mientras que la reputación exige un esfuerzo más integral y de acciones a lo largo del tiempo.

Palabras clave: Responsabilidad Social Empresarial, Imagen Corporativa, Reputación, Acción Social, Relaciones con la Comunidad.

JEL: M14

Abstract: This article is the result of a study to the city of Cartagena chain stores to measure the impact of programs of contributions and donations on the corporate image and reputation. First is a followup of the recent theoretical discussion around the concepts of CSR, contributions and donations, corporate image and reputation. Then applies a quantitative method and four scenarios are formulated to measure the incidence of variables social action, and relationships with the community about the corporate image and reputation. The theoretical discussion found that CSR should be understood as a philosophy of business management committed to the improvement of the living conditions of society in general. Additionally, hypothesis tests confirmed the conceptual differences between the corporate image and reputation for being a direct and positive relationship between the variables in social action and relations with the

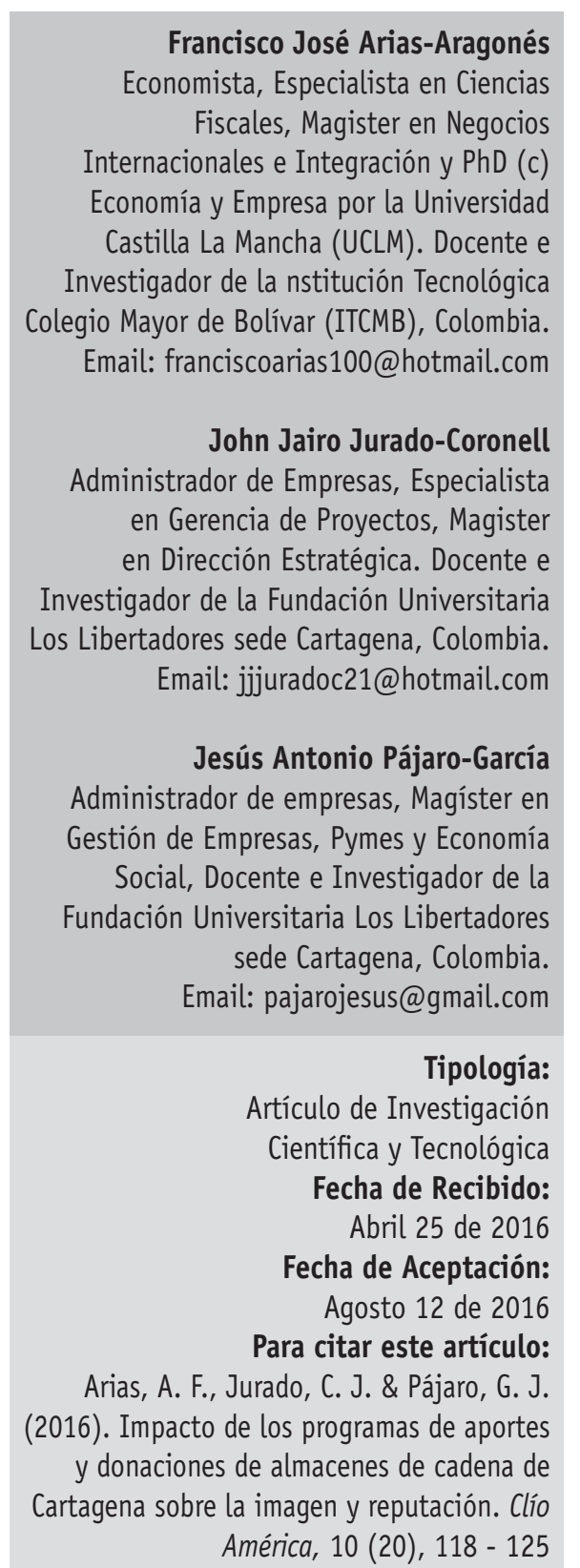


community about the corporate image and no direct relationship between the variables of social action and reputation. This shows that the contributions and donations programs can contribute to improve the corporate image, while reputation requires a more comprehensive effort and actions over time.

Keywords: Corporate Social Responsibility, Corporate Image, Reputation, Social Action, Community Relations.

\section{Introdución}

La Responsabilidad Social Empresarial (RSE) debe ser entendida como el compromiso que asume la organización empresarial con el desarrollo económico sostenible y con el mejoramiento de las condiciones de vida de la sociedad en general, lo cual va más allá del mero cumplimiento de las normas y las leyes. La RSE implica una gestión de la actividad empresarial con la capacidad de reconocer y retornarle a la sociedad beneficios en atención a la solución de variadas problemáticas tales como la conservación del medio ambiente, acceso a la salud, educación y reducción de brechas, entre otras. Lo anterior no es puramente motivado por el interés de captar una porción mayor de la torta de mercado, sino por ser la conducta correcta y aquella que garantiza el cumplimiento de los derechos universales de los diferentes sectores sociales (Feldman \& Reficco, 2015).

La sociedad de hoy demanda que las empresas asuman una conducta ética, responsable y comprometida con la solución de los efectos perniciosos que la actividad empresarial ejerce sobre los entornos social, ambiental, económico, político y cultural (Antelo-González \& Alfonso-Robaina, 2015). La sostenibilidad de la empresa moderna obliga a que éstas dejen de ser el problema y se conviertan en la solución, dado que la conservación del medio ambiente, la inversión en capital humano y el bienestar social son la base que asegura el éxito y continuidad de la actividad empresarial (Correa, 2004 y Sanclemente, 2015).

El avance y posicionamiento global de la RSE ha tenido mucho que ver con la erosión del antiguo paradigma, bajo el cual las empresas solo tenían que rendirle cuentas a sus accionistas o dueños, sin tener que responsabilizarse de ninguna acción respecto de sus demás grupos de interés o stakeholders. En los años 80's se inicia un cambio hacia una interpretación más amplia de la RSE por la creciente influencia de grupos de activistas; en virtud de eso, se empiezan a dar avances tendientes a la formalización de "políticas y sistemas de gestión que promuevan las relaciones con los accionistas, empleados, clientes, proveedores y medio ambiente" (Avendaño, 2013, p.155). En América Latina la RSE tuvo un vigoroso impulso hacia los años ochenta, debido a la mezcla variopinta de "factores económicos, sociales y políticos de largo plazo" (Ogliastri \& Reficco, 2009). La definición de RSE en la región no es única (Fondo Multilateral de Inversiones del Banco Interamericano de Desarrollo, 2009); siendo las más aceptadas la del Instituto Ethos de Brasil y la del Instituto Argentino de Responsabilidad Social Empresaria (IARSE). Ambas definiciones hacen énfasis en la ética, en el desarrollo sostenible, en el respeto por la diversidad y la reducción de brechas sociales. No obstante, la definición de RSE por la International Organization for Standarization (ISO), la cual llevó a la Norma ISO 26000 (2010), según Feldman \& Reficco (2015) es la más trabajada y consensuada a escala global. Adicionalmente, "la responsabilidad social es la acción estratégica visionada desde el pensamiento de los directivos" que alinea la actuación empresarial, teniendo en cuenta las implicaciones éticas que recaen sobre ellas (Mendoza, Salas \& López, 2015).

El concepto de RSE se encuentra sometido a permanente discusión por el rápido cambio en las expectativas de la sociedad y diferencias en la percepción de la publicidad por parte de los consumidores. Adicionalmente, la RSE se ha convertido en un enfoque cada vez más importante para la introducción de productos y el fortalecimiento de 
Impacto de los programas de aportes y donaciones de almacenes de cadena de Cartagena sobre la imagen y reputación

la imagen de marca a través de esa diferenciación (Dündar, 2015).

La imagen de marca y la reputación corporativa son conceptos sobre los cuales hay un gran debate centrado en si son conceptos cercanos o distantes (Smaiziene \& Jucevicius, 2009; Neville \& Bell, 2005; Cornelissen, 2004), discusión que no es objeto de este trabajo. La imagen de marca y reputación corporativa pueden ser adquiridas a través de la RSE, lo cual incluye la calidad de los productos, desempeño financiero, entorno social y servicios corporativos (Alsop, 2006). "Las reputaciones indican a los públicos cómo son los productos, los trabajos, las estrategias y las perspectivas de las compañías en comparación con sus competidoras" (Ruiz, Gutierrez \& Esteban, 2012, p. 10). La buena reputación es un valioso recurso que permite a las organizaciones capturar beneficios derivados de: cargar precios más elevados, reducir costos de transacción, atraer y contratar a los mejores trabajadores y fidelizar a sus clientes, entre otros. Adicionalmente, la imagen corporativa está relacionada con la impresión (positiva 0 negativa) que se forma una persona, luego de recibir una señal o mensaje de la compañía (Cornelissen, 2004). De ahí la importancia de la gestión de la imagen corporativa para que sea atractiva y genere valor entre los stakeholders (Limón, 2009).

De la filantropía corporativa se ha evolucionado a lo que hoy son los programas de aportes y donaciones y la inversión social. La importancia de lo anterior estriba en que las empresas pueden hacer cosas que una persona natural no puede; por ejemplo, una empresa puede movilizar recursos materiales y humanos, también puede poner horas de sus empleados a disposición de una Organización No Gubernamental (ONG), puede mejorar las condiciones de vida de una comunidad aledaña, entre muchas otras posibilidades. Estas acciones en muchos casos le representarían menos en dinero y esfuerzos pagar para que otro las hiciera que hacerlas por cuenta propia. La razón que soporta esto es que la articulación y sinergia de la empresa con el entorno no solo mejora la entrega de valor, sino que crea valor sostenible al hacerlo (Machado \& Gil, 2010). Adicionalmente, Machado \& Gil (2010) sostienen que los accionistas ejercen presión para que las donaciones estén relacionadas con la estrategia de negocios y contribuyan a generar un vínculo positivo con los clientes o grupos de interés relevantes. Entretanto, la sociedad reclama transparencia en las donaciones y pruebas de un verdadero compromiso con la problemática social abordada.

En este orden de ideas, las organizaciones con la capacidad de entender lo anterior son aquellas que logran ventaja competitiva por posicionarse como top of the heart entre sus stakeholders, tras lograr afectar positivamente la imagen corporativa y la reputación. Sin embargo, la RSE no debe convertirse en un acto de publicidad, ya que esto puede tener un efecto negativo sobre la reputación de la empresa (Dündar, 2015).

Los almacenes de cadena en la ciudad de Cartagena de Indias, al igual que muchas empresas en el resto del mundo, se están sumando al llamado internacional de generar un soporte socialmente responsable en todas las acciones, alineadas a su actividad principal y a la estrategia de negocio. Es así como vienen desarrollando estrategias de acercamiento a la comunidad, sobre todo a aquellas menos favorecidas, disponiendo para ellas algunos recursos que impactan en su calidad de vida, tal como sucede con mucha frecuencia en el sector de la salud (Arias, Caraballo \& Matos, 2012; Arias \& Diaz, 2015; Arias, Caraballo \& Matos, 2015; Arias, Caraballo \& Muñoz, 2016 y Arias, Matos y Caraballo, 2016)

A pesar de todas estas "buenas acciones" que los almacenes de cadena realizan para mantener buenas relaciones con sus grupos de interés, "cabe la posibilidad de que para los compradores el impacto no sea favorable y se vea perjudicada la imagen y reputación de la organización" (Murillo, 2014). La situación se complica aún más, debido a que algunos de estos aportes son realizados por los clientes en el momento de la compra, bien sea donando parte de las devoluciones por el pago o por la compra de donaciones simbólicas reconocidas con denominaciones específicas y ampliamente reconocidas. Estos aportes son entregados en nombre del almacén a las comunidades beneficiarias y no en nombre de los clientes, lo que podría tergiversar el concepto y apreciaciones sobre estos programas, impactando de manera desfavorable en la mente del cliente y la comunidad en general. 
Por lo anterior, es relevante mejorar la comprensión sobre la forma en la que impactan los programas de RSE desarrollados por los almacenes de cadena en Cartagena de Indias, basados en aportes y donaciones que efectúan los compradores, en la imagen corporativa y en la reputación, de acuerdo a las percepciones de los clientes y consumidores.

Para efectos de esta investigación, se entiende el programa de RSE desarrollado por los almacenes de cadena, solamente en su dimensión que cobija programas de beneficio a la comunidad y su impacto en la imagen corporativa, aislando los demás stakeholders que conforman el programa de RSE global. Según los indicadores Ethos (2007) la comunidad es uno de los stakeholders que demandan más atención por parte de la directiva empresarial; las dimensiones consideradas para su estudio son dos. La primera está asociada con las relaciones con la comunidad local, quien a su vez se subdivide en dos indicadores: administración del impacto de la compañía en la comunidad de entorno y relaciones con organizaciones locales. La segunda se denomina acción social y se subdivide en dos indicadores: financiamiento de la acción social e involucración con la acción social.

\section{Metodología}

El estudio es de tipo explicativo y utiliza el método cuantitativo para medir la incidencia de las variables acción social y relaciones con la comunidad local sobre la imagen corporativa y reputación de los almacenes de cadena de Cartagena. Para hacer eso, se formularon las siguientes hipótesis:

- Hipótesis I (H1). La variable acción social percibida por los clientes de los almacenes de cadena, incide directa y significativamente en la imagen corporativa.

- Hipótesis II (H2). La variable acción social percibida por los clientes de los almacenes de cadena, incide directamente en la reputación de la empresa.

- Hipótesis III (H3). La variable relaciones con la comunidad percibida por los clientes de los almacenes de cadena, incide directa y significativamente en la imagen corporativa.
- Hipótesis IV (H4). La variable relaciones con la comunidad percibida por los clientes de los almacenes de cadena, incide directa y significativamente en la reputación de la empresa.

El modelo teórico propuesto en el presente estudio se plantea en la figura 1; se basa en las hipótesis anteriores y se somete a prueba con la metodología propuesta. Adicionalmente, la tabla 1 muestra la distribución de la muestra y la tabla 2 corresponde a los detalles técnicos de la investigación.

\section{fFigura 1.}

\section{Modelo Teórico}

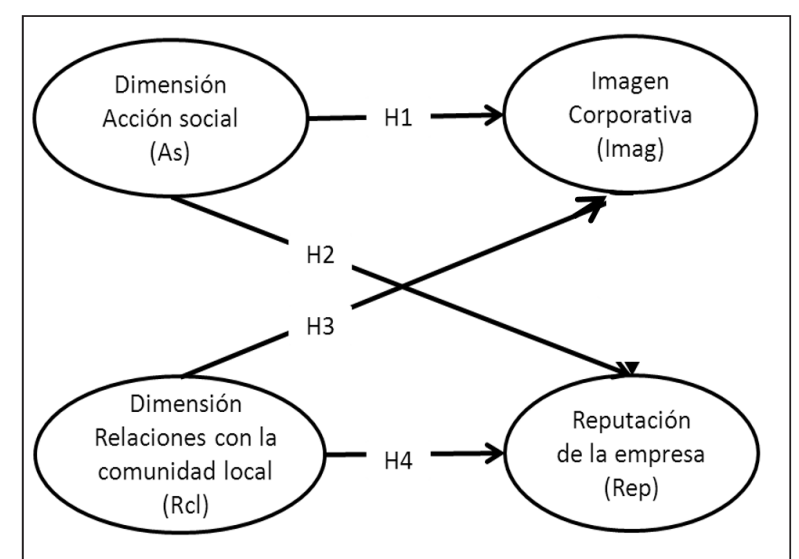

Fuente: elaboración propia.

Se diseñó una encuesta para obtener información y validar el modelo causal; la información fue recogida mediante un cuestionario estructurado con preguntas cerradas, elaboradas bajo la escala de Likert de 5 puntos. El cuestionario también recogió información sobre la imagen y la reputación, las cuales se miden a partir de la escala desarrollada por Ahearne, Bhattacharya \& Gruen (2005); la dimensión relaciones con la comunidad y acción social son desarrolladas a partir de Ethos (2007).

La siguiente es la distribución de la muestra: 
- Tabla 1.

\section{Distribución de la muestra}

\begin{tabular}{|l|c|c|}
\hline CONSUMIDORES & FRECUENCIAS & PORCENTAJES \\
\hline GÉNERO & & \\
\hline Hombres & 42 & 36,1 \\
\hline Mujeres & 85 & 63,9 \\
\hline FRECUENCIA DE COMPRAS EN ALMACENES DE CADENA & & \\
\hline Diario & 4 & 3,1 \\
\hline Semanal & 33 & 25,6 \\
\hline Quincenal & 57 & 44,2 \\
\hline Mensual & 35 & 27,1 \\
\hline
\end{tabular}

Fuente: elaboración propia con base a la encuesta de RSE en almacenes de Cartagena (2015)

Tabla 2.

\section{Detalles técnicos de la investigación}

\begin{tabular}{|l|l|}
\hline Ámbito Geográfico & Cartagena, Colombia \\
\hline Universo & $\begin{array}{l}\text { Compradores de almacenes de } \\
\text { cadena }\end{array}$ \\
\hline Metodologia & $\begin{array}{l}\text { Entrevista personal mediante } \\
\text { cuestionario estructurado }\end{array}$ \\
\hline $\begin{array}{l}\text { Procedimiento de } \\
\text { Muestreo }\end{array}$ & Por conveniencia \\
\hline Tamaño Muestral & 133 Encuestas \\
\hline
\end{tabular}

Fuente: elaboración propia con base a la encuesta de RSE en almacenes de Cartagena (2015).

\section{Resultados}

En la tabla 3 aparecen los resultados de la investigación los cuales muestran una relación directa entre algunas de las variables estudiadas, dado que la relación es directa al momento de aplicar las pruebas de correlación de los datos. Para el caso de la variable acción social, teniendo en cuenta el objetivo de la investigación, fueron seleccionadas las preguntas "conozco el destino de los recursos obtenidos por los almacenes de cadena mediante estas campañas" (P4) y "conozco los resultados que han tenido los recursos y acciones realizadas en las campañas de acción social de los almacenes de cadena" (P5), para la variable relaciones con la comunidad se seleccionó la pregunta "es una empresa consiente del impacto que tienen sus actividades en la comunidad de entorno"(P14), para la variable imagen se seleccionó la pregunta "es una empresa con responsabilidad social"(P16) y para la variable reputación la pregunta "es una empresa reconocida"(P23), aplicándole a estas relaciones la prueba de chi-cuadrado de Pearson para determinar el grado de relación entre las variables.

Los datos indican que existe una relación directa y positiva entre las variables acción social e imagen, de acuerdo a las pruebas de chi-cuadrado de Pearson el nivel de confianza entre las variables es de $95 \%$ y el nivel de significación es de $5 \%$, el $p$ - valor obtenido es mayor que 0,05 , ya que es 0,204 , resultado del análisis aplicado entre las preguntas P4 y P16, permitiendo afirmar que se cumple la hipótesis $\mathrm{H}$. El análisis de la relación entre las variables acción social y reputación, con las pruebas de los resultados de las preguntas P5 y P16, con un nivel de confianza del $95 \%$ y nivel de significación del $5 \%$, el $p$-valor obtenido es mayor que 0,05 , ya que es 0,731 , lo cual muestra que no existe una relación directa entre las variables y que por el contrario son independientes, conduciendo a rechazar la hipótesis $\mathrm{H} 2$.

Respecto a la relación entre las variables relaciones con la comunidad e imagen, los resultados indican 
que si existe una relación directa y positiva, las pruebas de chi-cuadrado entre las preguntas P14 y P16 presentaron que el nivel de confianza es de $95 \%$ y el nivel de significación es $5 \%$, el $p$ - valor obtenido es menor que 0,05 , ya que es 0,000 , lo cual conduce a aceptar la hipótesis $\mathrm{H} 3$, de igual forma se pudo determinar que existe una relación directa y positiva entre las variables relaciones con la comunidad y reputación teniendo en cuenta los resultados de las pruebas de chi-cuadrado entre las preguntas P14 y P23, las cuales indican que el nivel de confianza es de $95 \%$ y el nivel de significación es $5 \%$, el $p$-valor obtenido es menor que 0,05 , ya que es 0,032 , conduciendo a aceptar la hipótesis $\mathrm{H} 4$.

ITabla 3.

\section{Pruebas de Chi cuadrado de Pearson}

\begin{tabular}{|c|c|c|c|c|c|c|}
\hline \multicolumn{7}{|c|}{ ANALISIS DE TABLAS CRUZADAS ENTRE VARIABLES } \\
\hline Variables & Hipótesis & Análisis & Valor & gl & $\begin{array}{l}\text { Sig. asintótica } \\
\text { (bilateral) }\end{array}$ & $\begin{array}{c}\text { Validez/rechazo } \\
\text { Hipótesis }\end{array}$ \\
\hline \multirow{4}{*}{$\begin{array}{l}\text { Acción Social e } \\
\text { Imagen }\end{array}$} & \multirow{4}{*}{$\mathrm{H} 1$} & Chi-cuadrado de Pearson & $20,363^{\mathrm{a}}$ & 16 & ,204 & \multirow{4}{*}{ Se acepta } \\
\hline & & Razón de verosimilitudes & 20,357 & 16 & ,205 & \\
\hline & & Asociación lineal por lineal & 875 & 1 &, 350 & \\
\hline & & $\mathrm{N}$ de casos válidos & 132 & & & \\
\hline \multirow{4}{*}{$\begin{array}{l}\text { Acción Social y } \\
\text { Reputación }\end{array}$} & \multirow{4}{*}{$\mathrm{H} 2$} & Chi-cuadrado de Pearson & $12,191^{\mathrm{a}}$ & 16 & ,731 & \multirow{4}{*}{ Se rechaza } \\
\hline & & Razón de verosimilitudes & 14,931 & 16 &, 530 & \\
\hline & & Asociación lineal por lineal &, 661 & 1 &, 416 & \\
\hline & & N de casos válidos & 128 & & & \\
\hline \multirow{4}{*}{$\begin{array}{l}\text { Relaciones con } \\
\text { la Comunidad e } \\
\text { Imagen }\end{array}$} & \multirow{4}{*}{ H3 } & Chi-cuadrado de Pearson & $69,943^{\mathrm{a}}$ & 16 &, 000 & \multirow{4}{*}{ Se acepta } \\
\hline & & Razón de verosimilitudes & 64,147 & 16 &, 000 & \\
\hline & & Asociación lineal por lineal & 31,545 & 1 &, 000 & \\
\hline & & $\mathrm{N}$ de casos válidos & 131 & & & \\
\hline \multirow{4}{*}{$\begin{array}{l}\text { Relaciones con } \\
\text { la Comunidad y } \\
\text { Reputación }\end{array}$} & \multirow{4}{*}{$\mathrm{H} 4$} & Chi-cuadrado de Pearson & $27,905^{\mathrm{a}}$ & 16 &, 032 & \multirow{4}{*}{ Se acepta } \\
\hline & & Razón de verosimilitudes & 29,188 & 16 &, 023 & \\
\hline & & Asociación lineal por lineal & 12,920 & 1 &, 000 & \\
\hline & & $\mathrm{N}$ de casos válidos & 126 & & & \\
\hline
\end{tabular}

Fuente: elaboración propia con base a la encuesta de RSE en almacenes de Cartagena y el software SPSS.

Los resultados del presente estudio guardan relación con lo encontrado por Cornelissen (2004), quien relaciona la imagen corporativa con las impresiones espontáneas de una persona luego de recibir una señal o mensaje de la compañía. Esto se evidencia en la relación directa y positiva entre las variables acción social, relaciones con la comunidad e imagen corporativa, que condujeron a la aceptación de las hipótesis 1 y 3 ( $\mathrm{H} 1$ y H3). Lo anterior deja ver que los programas de aportes y donaciones tienen la capacidad de mejorar la imagen corporativa. Por otra parte, el rechazo de la hipótesis 2 ( $\mathrm{H} 2)$ se relaciona con el hecho de que la reputación es el resultado de la evaluación global de los procesos y resultados de la compañía por parte de los grupos de interés (Neville \& Bell, 2005), lo cual "sería un concepto más profundo que se desarrolla con el tiempo como consecuencia de las acciones, conductas y comunicaciones de la organización" (Ruiz, Gutiérrez \& Esteban, 2012), cosa que sería difícil de lograr solamente con un programa de aportes y donaciones en el marco actividades de RSE. Sin embargo, no se 
Impacto de los programas de aportes y donaciones de almacenes de cadena de Cartagena sobre la imagen y reputación

descarta la posibilidad de que la sostenibilidad en el tiempo de un programa de aportes y donaciones pueda impactar favorablemente en la reputación corporativa, lo cual sería objeto de estudio de futuras investigaciones.

\section{Conclusión}

La discusión teórica en el tema de la RSE enfatizando en los programas de aportes y donaciones, sumado con el abordaje de los conceptos de imagen corporativa y reputación, al ser confrontados con los resultados cuantitativos de la presente investigación permite llegar a varias conclusiones y reflexiones que ayudan a mejorar la comprensión del impacto de estos programas sobre la imagen corporativa y reputación desde una postura crítica frente a la problemática investigada.

El estudio muestra que la RSE debe ser una filosofía presente en la gestión de la actividad empresarial, comprometida con el desarrollo económico sostenible y con el mejoramiento de las condiciones de vida de la sociedad en general, lo cual trasciende el mero cumplimiento de las normas y las leyes. Los aportes y donaciones, los cuales forman parte de los programas de RSE tienen un mayor alcance que las donaciones realizadas las personas naturales. Sin embargo, estos programas no deben convertirse en un acto de publicidad, puesto que la sociedad reclama transparencia en las donaciones y pruebas de un verdadero compromiso con la problemática social abordada.

Los resultados de las pruebas de hipótesis confirmaron diferencias conceptuales entre la imagen corporativa y reputación planteadas por diferentes estudios; aunque esa discusión conceptual no haya sido el objetivo principal de la investigación resultó siendo un hallazgo importante en el sentido de que la relación directa y positiva de las variables acción social y relaciones con la comunidad sobre la imagen corporativa, permite mostrar que los programas de aportes y donaciones pueden contribuir a mejorar la imagen corporativa por mandar un mensaje positivo a los grupos de interés o stakeholders. En el caso de la dimensión acción social se encontró que no existe una relación directa con la reputación y son variables independientes, lo cual llevó a rechazar la hipótesis $2(\mathrm{H} 2)$. Dicho resultado es consistente con los de varias investigaciones que consideran la reputación como un concepto más profundo que se desarrolla con el tiempo como resultado de las acciones, conductas y comunicaciones de la organización. De conformidad con lo anterior, el estudio ha mostrado que los programas de aportes y donaciones pueden contribuir mejorar la imagen corporativa, mientras que el fortalecimiento de la reputación exige un esfuerzo más integral y con acciones a lo largo del tiempo, lo cual ratifica los resultados de varias investigaciones influyentes en el tema.

Las conclusiones a las cuales se ha llegado tienen validez solamente para los almacenes de cadena de la ciudad de Cartagena. Se hace necesario que se estudien estas mismas variables en otras ciudades de Colombia y en otros sectores de la economía, con la finalidad de confirmar o refutar las conclusiones del presente trabajo.

\section{Referencias Bibliográficas}

Ahearne, M., Bhattacharya, C. B., \& Gruen, T. (2005). Antecedents and consequences of customer-company identification: expanding the role of relationship marketing. Journal of Applied Psychology, 90 (3), 574

Alsop, Ronald J. (2006). Business Ethics Education in Business Schools: A Commentary. Journal of Management Education, Vol. 30 (1), 11-14.

Antelo-González, Y. Y., \& Alfonso-Robaina, D. (2015). Análisis de la Responsabilidad Social Empresarial Basado en un Modelo de Lógica Difusa Compensatoria. Ingeniería Industrial, 36 (1), 58-69. Recuperado de: http://scielo.sld.cu/scielo.php?script=sci_arttext\&pid $=$ S1815-59362015000100007

Arias, F. J. A \& Díaz, K. L.J. (2015). El turismo médico en Cartagena de indias: directrices para su desarrollo. Méthodos 13, 57-72.

Arias, F. J. A., Caraballo, A. M. P., \& Matos, R. E. N. (2012). El turismo de salud: Conceptualización, historia, desarrollo y estado actual del mercado global. Clío América, 6 (11), 72-98.

Arias, F. J. A., Caraballo, A. M. P., \& Matos, R. E. N. (2015). La Oferta de Turismo Médico en la Ciudad de Cartagena. Revista Sotavento MBA, Vol. (25), 10-17. 
Arias, F.J., Caraballo, A.M \& Muñoz, J. M. (2016), El turismo médico en Cartagena: "oferta y barreras". Dimensión Empresarial, 14 (2), 145-165. doi.org/10.15665/rde. v14i2.457

Arias, F. J. A, Matos, R.E.N \& Caraballo, A. M.P. (2016). Turismo médico: Caracterización de su oferta en el Distrito de Cartagena. Cartagena de Indias: Editorial Bonaventuriana.

Avendaño, W. R. (2013). Responsabilidad social (RS) y responsabilidad social corporativa (RSC): una nueva perspectiva para las empresas. Revista lasallista de investigación, 10 (1), 152-63.

Banco Interamericano de Desarrollo (BID) y Fondo Multilateral de Inversiones (FOMIN). (2009). Guía de aprendizaje sobre la implementación de responsabilidad social empresarial en pequeñas y medianas empresas. Recuperado de: http://idbdocs.iadb.org/wsdocs/getdocument. aspx?docnum $=35025627$

Cornelissen, J.P. (Ed.). (2004). Corporate Communications: Theory and Practice. Londres: Sage Publications.

Correa, M. E. (2004). Responsabilidad social empresarial: una nueva forma de hacer negocios. Revista Futuros, 6 (2), 3-4.

Dündar, F. N. (2015). Influence of corporate social responsibility on corporate image and reputation. Proceedings of the multidisciplinary academic conference. Recuperado de: http://connection.ebscohost.com/c/articles/111482191/ influence-corporate-social-responsibility-corporate-imagereputation.

Feldman, P. M., \& Reficco, E. (2015). Impacto de la responsabilidad social empresarial en el comportamiento de compra y disposición a pagar de consumidores bogotanos. Estudios Gerenciales, 31 (137), 373-382.

ISO (2010). ISO 26000:2010 Guidance on social responsibility. Recuperado de: http://www.iso.org/iso/home/store/catalogue_tc/catalogue_detail.htm?csnumber=42546.

Limón, M. (Ed.). (2008). Imagen corporativa. Estrategia organiza- cional de comunicación Global. México. Editorial Trillas.
Machado, F., \& Gil, M. (2010). Donación empresarial: de la filantropía a la inversión social. Santiago: Centro de Estudios de emprendimientos solidarios (CEES-UC). Recuperado de http://www.superacionpobreza.cl/wp-content/ uploads/2014/03/donacion_empresarial.pdf

Mendoza, F. D., Salas, S. E., \& López, J. D. (2015). Responsabilidad social en Universidades Públicas Autónomas: Esencia académica con visión transformadora. Clio América. 9 (18), 135 - 142

Murillo, M., G. (2014). Relación entre las estrategias de responsabilidad social empresarial aplicadas por la IPS calidad humana y su imagen y reputación. Recuperado de: http:// repository.unimilitar.edu.co/bitstream/10654/12515/1/ ARTICUL0\%20\%20FINAL\%20UNI\%20MILITAR.pdf?

Neville, B.A. \& Bell, S.J. (2005). Corporate reputation, stakeholders and the social performance-financial performance relationship. European Journal of Marketing: 39(9/10), 1184-1195.

Ogliastri, E. \& Reficco, E. (2009). Empresa y sociedad en América Latina: una introducción. Academia Revista Latinoamericana de Administración, 43, 1-25.

Oliveira, N. V., \& Smith, V. (2007). Indicadores Ethos de responsabilidad social empresarial. Recuperado de http:// www1.ethos.org.br/ EthosWeb/arquivo/0-A-bbe2011_ Indic_ETHOS_ESP.pdf

Ruiz, B., Gutiérrez, S., \& Esteban, Á. (2012). Desarrollo de un concepto de reputación corporativa adaptado a las necesidades de la gestión empresarial. Strategy \& Management Business Review, 3(1), 9-31.

Sanclemente, T. J. (2015). La responsabilidad social empresarial (RSE) de los pequeños distribuidores minoristas, Clío América, 9 (17), 42 - 56

Smaiziene, I. \& Jucevicius, R., (2009); Corporate reputation: Multidisciplinary Richness and Search for relevant Definition. Inzinerine Ekonomika-Engineering Economic, 2, 91-100 